\title{
Aplicação de redes neurais ART e análise de textura para a identificação do estado de alteração de agregados minerais
}

\author{
Luciano José Senger ${ }^{1}$ \\ Lilian Taís de Gouveia ${ }^{2}$
}

\begin{abstract}
Resumo: Uma nova abordagem para identificação do estado de alteração de agregados minerais destinados à obras de construção civil é apresentada. Tal identificação é de fundamental importância para evitar insucessos e ocorrência de defeitos prematuros na realização de obras que podem ser atribuídos à qualidade do agregado utilizado quanto ao seu estado de alteração. Técnicas de processamento de imagens são empregadas para aquisição dos histogramas dos canais de cor das imagens, seguidos do cálculo da entropia dos histogramas que fornece as características principais para a classificação. Finalmente, um modelo de aquisição de conhecimento incremental e de classificação que emprega redes neurais ART (Adaptive Resonance Theory) é construído para automatizar o processo de classificação. O modelo de classificação é organizado em duas etapas. Na primeira etapa, os agregados são classificados como alterados e não alterados, e em uma segunda etapa, o grupo de agregados alterados é classificado quanto ao grau de alteração. O modelo proposto apresenta resultados de classificação melhores quando comparados com aqueles obtidos através de outros algoritmos de classificação.
\end{abstract}

Palavras-chave: redes neurais, análise de textura, processamento de imagens

\begin{abstract}
A new approach to identify weathered and non-weathered aggregates that will be employed in construction works is presented. This identification is important to prevent premature failures and defects in works that can be attributed to the use of weathered aggregates. Image processing techniques are employed to acquire the color histograms, followed by calculating the entropy of the histograms which provides the main features for classification. Finally, a model of incremental acquisition of knowledge and classification that uses ART (Adaptive Resonance Theory) neural networks is built to automate the process of classification. This model is built through two steps. In the first step, the aggregates are classified as weathered and non-weathered, and in a second step, the group of weathered aggregates are classified on its weathering level. The proposed model presents better classification results when compared with the ones obtained through other classification algorithms.
\end{abstract}

\footnotetext{
${ }^{1}$ Departamento de Informática, UEPG, PR, Brasil. 1 jsenger@uepg . br

${ }^{2}$ Departamento de Engenharia Civil, UEPG, PR, Brasil. Itgouveia@gmail.com
} 
Keywords: neural networks, texture analysis, image processing

\section{Introdução}

$\mathrm{O}$ agregado mineral (fragmento de rocha) é utilizado como constituinte principal de muitos materiais utilizados para construção, incluindo concreto de cimento Portland, concreto asfáltico, bases de rodovias, lastros de ferrovias, enrocamentos, drenos e sistemas de filtragem. Em vista disto, milhões de toneladas de agregados minerais são produzidas e consumidas pela indústria da construção civil anualmente [19,21].

Os agregados devem atender à requisitos de qualidade com base nas especificações técnicas necessárias para o fim ao qual se destina. Esses requisitos estão diretamente relacionados às adequadas propriedades desses materiais. Propriedades físicas como resistência à compressão e à abrasão, necessárias para praticamente todos os propósitos de construção, são propriedades determinadas principalmente pelas características da rocha de origem. Entretanto, o processo de produção em pedreiras pode afetar significativamente a qualidade do agregado com a eliminação de camadas de rochas fracas ou alteradas que freqüentemente encontram-se em meio à camadas de rochas sãs [26].

Muitos casos de insucessos e ocorrência de defeitos prematuros na realização de obras podem ser atribuídos à qualidade do agregado utilizado quanto ao seu estado de alteração $[18,24,30]$. Portanto, é de interesse das pedreiras que realizam a extração de rochas a detecção imediata da presença de materiais alterados, de modo a garantir sempre a qualidade do agregado fornecido. Assim também é de interesse de engenheiros e construtores de obras o conhecimento do estado de alteração dos agregados, para o não comprometimento das obras.

$\mathrm{O}$ processo de alteração se inicia quando as rochas são expostas à condições ambientais diferentes daquelas onde se formaram, em contato com novos processos físicos e químicos, que diferem em muito das condições originais. Processos de intemperismo físico criam micro e macro fraturas nas rochas que vão facilitar a infiltração de soluções aquosas e a ação de microorganismos vivos, iniciando-se assim, uma série de reações químicas que variam em função das condições ambientais e da composição mineralógica das rochas [22]. Esses processos de alteração podem afetar a resistência mecânica e durabilidade do agregado, e, desta maneira, vir a comprometer a durabilidade e resistência mecânica das estruturas por ele formadas.

Geralmente diferentes processos de intemperização das rochas agem simultaneamente ou subseqüentemente sobre a superfície rochosa e apenas seus efeitos integrais podem ser vistos na superfície [2]. É importante notar que o processo de intemperização resulta em vários efeitos visíveis relacionados à aparência de cores dos agregados, como descoloração, manchas ferruginosas, escurecimento superficial, ou seja, esses materiais alterados passam a 
apresentar em sua superfície cores diferentes daquelas condizentes à sua condição mineralógica original [13].

São muitos os testes disponíveis para avaliação das condições de alteração dos materiais rochosos, entre eles inclui-se a análises petrográficas além de ensaios mecânicos para avaliações empíricas, com o intuito de detectar, através da diminuição da resistência mecânica e da durabilidade dos agregados, um possível processo de alteração das rochas [3]. No entanto, esses são ensaios laboratoriais que demandam uma grande quantidade de tempo e que devem ser realizados em laboratório apropriado por técnicos especializados. Portanto, seria impossível utilizá-los para detecção rápida de agregados alterados, por exemplo numa pedreira, onde tudo ocorre simultaneamente, a extração da rocha, transporte, britagens e estocagem.

Este trabalho apresenta um método rápido de detecção imediata de agregados alterados, sinalizada por sua textura e alteração de cores. Não foi considerado o tipo de alteração sofrida pelo material e nem os minerais resultantes dessa alteração, pois, do ponto de vista construtivo, não interessam os fatores que provocam a alteração, mas sim reconhecer se o material está ou não alterado, por este ser um forte indício de perda de resistência mecânica e de durabilidade desse material. O método proposto é de especial importância para as pedreiras que precisam imediatamente detectar quando uma camada de material alterado foi alcançada, além de servir como um método menos detalhado, porém rápido, de detecção de agregados alterados e seu grau de alteração. Além disso, este método pode ser utilizado de maneira conjunta à outros ensaios já consagrados, por exemplo ensaios mecânicos, como uma maneira simples e eficiente de sinalização do processo de alteração. Portanto, este trabalho traz uma abordagem de caracterização e classificação automática baseada na análise de imagens de texturas das partículas de agregados com diferentes níveis de alteração.

\section{Aquisição das Imagens das Partículas de Agregados}

Seis agregados basálticos foram considerados nesse estudo, sendo três deles agregados sem nenhum tipo de alteração e outros três alterados em diferentes níveis. Um total de 600 partículas (amostras de agregados) foram analisadas, sendo 100 para cada amostra. As partículas foram dispostas sobre o scanner de maneira que repousassem sobre seu eixo de maior estabilidade, para a aquisição das imagens. As imagens foram obtidas com resolução de 300 dpi em um ambiente completamente escuro, evitando, dessa maneira, a interferência de outras fontes de luz.

Após a aquisição das imagens, estas foram pré-processadas para a eliminação de ruídos e transformadas em imagens binárias, com remoção de eventuais pequenos pontos isolados ao longo da superfície da imagem. Métodos de morfologia matemática foram utilizados para esta finalidade $[9,10]$. Obtida a imagem binária, o próximo passo foi a determinação 
do contorno de cada imagem através do border following method [9], que retorna uma lista com as posições $x$ e $y$ ao longo da borda. A detecção da borda permite obter informações de tons apenas para os agregados, desconsiderando a imagem de fundo (background). Tais informações são empregadas para a geração e análise dos histogramas da imagem.

\section{Histograma e Equalização de Imagens}

A técnica de tratamento de imagens por histogramas associa um valor $h(n)$ para cada nível de cinza $n$ da imagem. Os valores $h($.$) correspondem ao número de vezes, ou$ freqüência, que os tons de cinza ocorrem na imagem. Sendo uma imagem digital $g$ de tamanho $P \times Q(g(i, j)$, onde cada pixel apresenta um nível de cinza no intervalo $0 \leq n<N$ e $N$ o número total de níveis de cinza e igual a 255, o histograma $h(n) \leftarrow g(p, q)$ para cada pixel da imagem $(0 \leq p<P$ e $0 \leq q<Q)$ foi obtido através da Equação 1:

$$
h(g(p, q))=h(g(p, q))+1
$$

onde $g(p, q)$ representa o nível de cinza da imagem digital.

Neste estudo, foram considerados os histogramas obtidos pela imagem original, com informação de cores, e os histogramas em tons de cinza. Para cada imagem original, em cores, foram obtidos três histogramas, um para cada componente $R G B$. Similarmente os histogramas para cada componente das imagens coloridas de tamanho $P \times Q$ foram obtidos através da Equação 2:

$$
h_{c}\left(g_{c}(p, q)\right)=h_{c}\left(g_{c}(p, q)\right)+1
$$

onde $0 \leq c<3$ representa os componentes $R G B$ da imagem e $g_{c}($.$) correspondem$ aos três histogramas da imagem colorida. A partir dos histogramas foram obtidos os histogramas de frequiência relativa $(0 \leq i<N)$, para níveis de cinza e para a imagem colorida.

Os histogramas de freqüência relativa foram utilizados para realizar a equalização das imagens. A operação de equalização é descrita como um mapeamento $f: n \leftarrow f(n)$, onde a função $f(n)$ é incrementalmente construída a partir dos histogramas de freqüência relativa $h(n)$ (Equação 3):

$$
f(n)=(N-1) \sum_{k=0}^{n} h(k)
$$

A equalização por histograma é útil para aumentar ou normalizar o contraste das imagens para análises posteriores, de forma que a partir da imagem original $g(p, q)$ é obtida uma nova imagem $l(p, q)$ onde cada pixel corresponde à (Equação 4):

$$
l(p, q) \leftarrow f(g(p, q))
$$




\section{Entropia}

A medida entropia ou incerteza mede a quantidade de informação transferida por um canal ou gerada por uma fonte. Portanto, quanto maior o valor da entropia, mais informação estará associada ao canal [23]. O conceito de entropia aplicado à análise de imagens define que a geração de informação pode ser modelada como um processo probabilístico. Uma imagem é considerada como um processo aleatório sendo $p_{i}$ a probabilidade de um pixel $i$ da imagem assumir um valor entre 0 e $N$. A distribuição dos níveis de intensidade pode ser descrita por uma função de densidade de probabilidade, através da Equação 5:

$$
p(i)=\frac{h(i)}{P * Q}
$$

onde $h(i)$ corresponde ao histograma de freqüência da imagem, com $0 \leq i<N$, de forma que $\sum_{i=0}^{N} p(i)=1$.

A Entropia de Shanon [29] é uma medida de aleatoriedade estatística que pode ser usada para caracterizar a textura da imagem, calculada através da Equação 6:

$$
E=-\sum_{i=0}^{N} p(i) \ln p(i)
$$

onde $p_{i}(a)$ é a freqüência relativa do $i$-ésimo pixel de um imagem. $\mathrm{O}$ menor valor que pode ser obtido para $E$ é igual a zero, que corresponde a uma imagem na qual todos os pixels tem a mesma intensidade de tons. O maior valor que pode ser obtido corresponde a uma imagem que contém a mesma quantidade de pixels para todas as intensidades. A entropia não mede a disposição espacial da informação. Imagens com uma mesma quantidade de pixels e intensidade apresentam valores iguais de entropia, mesmo que a distribuição dos pixels na imagem seja diferente, como mostra a Figura 1. Pode-se observar através desse exemplo que o valor de entropia é o mesmo se a imagem for rotacionada ou transladada.

\section{Resultados e Análise dos Resultados}

Na Tabela 1 estão apresentadas as médias e as variâncias da entropia das imagens em escala de cinza e equalizadas, para as amostras não alteradas $b, b r$ e $b f$ (Figura 2) e alteradas $b 5, b a$ e be (Figura 3), respectivamente. As médias das entropias foram analisadas através do método estatístico de Análise de Variância (ANOVA) considerando um nível de confiança de $95 \%$. A análise de variância dos dados mostram se existe diferença entre as médias, sendo estas diferenças descritas pelo valor de $F$, obtido usando a metodologia ANOVA. O valor obtido de $F$ é de 75,92 , o qual é maior que o valor crítico $(2,23)$. Portanto, pode-se afirmar 


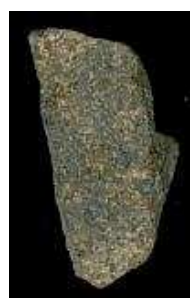

(a) $\mathrm{E}=4,648$

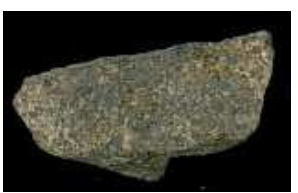

(b) $\mathrm{E}=4,648$

Figura 1. Exemplos de imagens com o mesmo valor de entropia $(E)$, sendo (b) rotacionada em 90 graus em relação a (a)

com $95 \%$ de confiança que as médias de entropia são significativamente diferentes entre os grupos.

O teste de Tukey [27] é usado geralmente em conjunção com o ANOVA de maneira a determinar quais grupos diferem significativamente dos outros. Nesse teste é obtido o valor de $q_{s}$ :

$$
q_{s}=\frac{Y_{a}-Y_{b}}{S},
$$

onde $Y_{a}$ e $Y_{b}$ correspondem, respectivamente, ao maior e ao menor valor entre as duas médias que estão sendo comparadas e $S$ é o erro padrão dos dados em questão. Se o valor de $q_{s}$ for maior que o valor crítico $q_{c}$ (o valor crítico é obtido em uma tabela estatística em função do grau de liberdade, do nível de significância e do número de grupos [27]), então os valores entre médias relativas aos grupos são ditas significativamente diferentes. $\mathrm{O}$ valor de $q_{c}$ obtido para este experimento foi de 4,29 .

O teste pareado entre os agregados $b$ e $b a$ resultou num valor de $q_{s}$ de 4,18 e entre $b f$ e $b a, q_{s}=3,93$. Portanto, $q_{s}<q_{c}$, o que indica que os agregados $b$ e $b f$ (não alterados) não foram diferenciados do agregado $b a$ (alterado). Para os outros pares de agregados foi obtido $q_{s}>q_{c}$. Os resultados indicam que as imagens em níveis de cinza e equalizadas não foram satisfatórias para promover a diferenciação entre agregados alterados e não alterados, pois não houve diferenciação entre o agregado $b a$ (alterado) dos agregados não alterados. Isso se deve, provavelmente ao tipo de alteração ocorrida no agregado $b a$ onde sobre suas partículas havia uma camada grossa de pó não removível com simples lavagem do material (Figura 7(b)). Camada esta que, em escala de cinza, camufla o estado de alteração do agregado. Portanto, o método de entropia, utilizando imagens em escala de cinza e equalizadas, não foi totalmente sensível para diferenciar todos os agregados alterados dos não alterados. 
Tabela 1. Entropia das imagens em escala de cinza e equalizadas

\begin{tabular}{c|c|c|c|c|c|c}
\hline agregados & b & br & bf & b5 & ba & be \\
\hline médias & 4,304 & 4,183 & 4,209 & 4,456 & 4,255 & 4,406 \\
variâncias & 35,711 & 9,243 & 16,670 & 3,831 & 4,608 & 9,834 \\
\hline
\end{tabular}

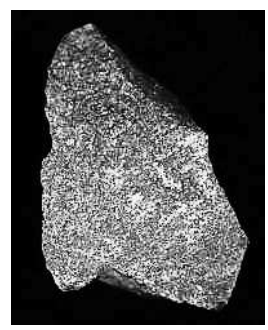

(a) $\mathrm{b}$

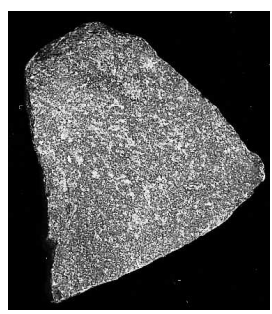

(b) br

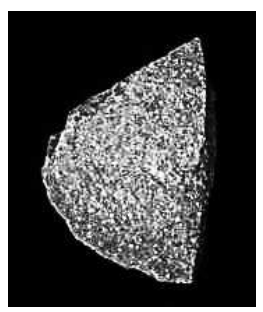

(c) bf

Figura 2. Exemplos de imagens em escala de cinza e equalizadas de partículas representativas das amostras basálticas sem alteração

Então, o próximo passo foi a análise da entropia das imagens coloridas, levando-se em conta as versão das imagens originais e equalizadas. Inicialmente, foi aplicada a equalização de modo a realçar detalhes difíceis de serem detectado nas imagens originais.

Na Tabela 2 estão apresentados os resultados médios das entropias para as componentes red $(E r)$, green $(E g)$ e blue $(E b)$, assim como o valor médio da entropia total (correspondente aos três componentes) e sua variância. Exemplos de imagens coloridas e equalizadas dos agregados não alterados e alterados estão apresentados nas Figuras 4 e 5 , respectivamente.

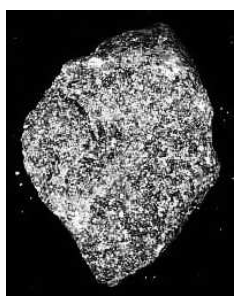

(a) b5

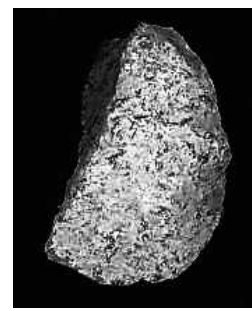

(b) ba

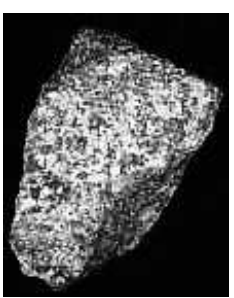

(c) be

Figura 3. Exemplos de imagens em escala de cinza e equalizadas de partículas representativas das amostras basálticas alteradas 
A análise de variância mostrou que existe diferenças entre as médias de entropia. $\mathrm{O}$ valor de $F$ obtido foi de 63,86 , valor este maior que o valor crítico $(2,24)$, para $95 \%$ de confiança. Portanto, pode-se afirmar que os valores de entropia diferem significativamente entre grupos. Para o agregado $b r$ e $b a$ foi obtido $q_{s}=1,97$ e para $b r$ e $b e, q_{s}=1,98$, os quais são menores que $q_{c}=4,29$. Para os outros pares de agregados, $q_{s}>q_{c}$. Portanto, o método da entropia com uso de imagens coloridas e equalizadas não foi sensível o suficiente para diferenciar todos agregados alterados dos não alterados. O resultado da equalização foi satisfatório do ponto de vista de realce, como pode ser visto nas imagens apresentadas nas Figuras 4 e 5, contudo aproximou muito as características de textura entre todos os agregados alterados e não alterados, dificultando a diferenciação entre eles.

Tabela 2. Entropia das imagens em cores e equalizadas

\begin{tabular}{c|ccc|c|c}
\hline & \multicolumn{3}{|c|}{ médias } & média total & variância \\
\hline agregados & $\mathrm{Er}$ & $\mathrm{Eg}$ & $\mathrm{Eb}$ & $\mathrm{Ergb}$ & var \\
\hline b & 4,296 & 4,309 & 4,282 & 4,296 & 40,379 \\
br & 4,421 & 4,417 & 4,402 & 4,413 & 08,925 \\
bf & 4,271 & 4,279 & 4,261 & 4,270 & 19,174 \\
b5 & 4,678 & 4,660 & 4,595 & 4,644 & 03,218 \\
ba & 4,473 & 4,459 & 4,407 & 4,446 & 04,455 \\
be & 4,479 & 4,456 & 4,404 & 4,446 & 09,591 \\
\hline
\end{tabular}

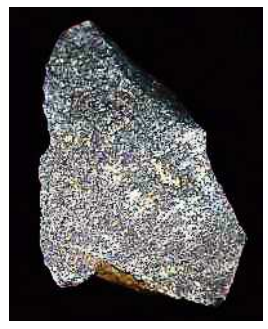

(a) b

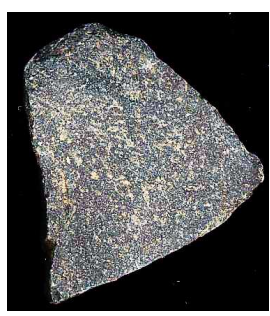

(b) br

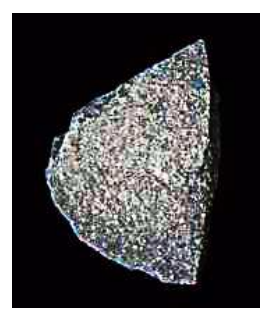

(c) bf

Figura 4. Exemplos de imagens coloridas e equalizadas de partículas representativas das amostras basálticas sem alteração

Visto que a entropia usando imagens em escala de cinza e equalizadas e imagens coloridas e equalizadas não diferenciou todos os agregados alterados dos não alterados, então, para a próxima análise foram usadas apenas as imagens coloridas (originais) sem equalização. Os resultados assim obtidos estão apresentados na Tabela 3. A análise de variância dos dados mostrou que há diferenças entre os valores médios de entropia. O valor de $F=79,67$ é maior que o valor crítico $(2,24)$ para $95 \%$ de confiança. Ao comparar todos os grupos verificou-se 


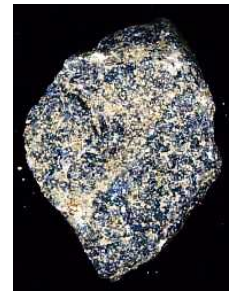

(a) b5

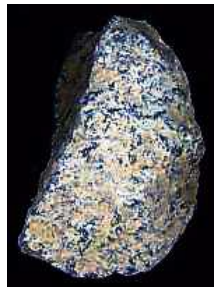

(b) ba

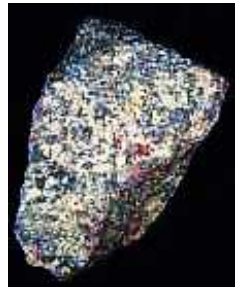

(c) be

Figura 5. Exemplos de imagens coloridas e equalizadas de partículas representativas das amostras basálticas alteradas

que $q_{s}<q_{c}$ para todos os agregados de mesma categoria. Portanto, os valores de entropia obtidos a partir das imagens coloridas sem equalização foi capaz de diferenciar todos os agregados alterados daqueles não alterados.

Pode-se ver, na Tabela 3, que os valores obtidos para $E g$ foram mais elevados que os valores de $E r$ e $E b$ para todos os agregados não alterados. Os agregados dentro da classe dos não alterados tiveram os valores de entropia total entre 4, 350 e 4, 450 aproximadamente, com pequenas variações em suas parciais $E r, E g$, Eb. A classe de agregados alterados, com valores de entropia acima de 4,450, tiveram uma subdivisão dentro da própria classe. Os agregados $b a$ e be, com valores de entropia entre 4,450 e 4,600 aproximadamente, apresentaram níveis de alteração semelhantes. O agregado com maior nível de alteração (b5), foi o que apresentou valores mais elevados de entropia, entre 4, 650 e 4,800. É interessante notar que a classe de agregados alterados apresentou acentuada diminuição da entropia da componente blue $(E b)$ e elevação da entropia da componente red $(E r)$ em relação à classe dos agregados não alterados. Esta característica sugere que o maior nível de alteração do agregado está associado à elevação de sua componente $E r$ e diminuição de sua componente Eb.

Em resumo, os resultados obtidos sugerem que as informações de cores estão fundamentalmente associadas ao nível de alteração dos agregados e as imagens coloridas sem equalização permitem a melhor separação entre agregados alterados e não alterados.

\section{Classificação Automática do Estado de Alteração dos Agregados}

Como visto, a entropia diferencia o estado de alteração dos agregados permitindo sua classificação. Portanto, considerando que há uma variedade de agregados com estados de alteração potencialmente diferentes, é adequada a definição e utilização de um modelo de 
Tabela 3. Entropia das imagens em cores e sem equalização

\begin{tabular}{c|ccc|c|c}
\hline & \multicolumn{3}{|c|}{ médias } & média total & variância \\
\hline agregados & Er & Eg & Eb & Ergb & var \\
\hline b & 4,385 & 4,407 & 4,373 & 4,388 & 38,457 \\
br & 4,406 & 4,409 & 4,388 & 4,401 & 08,628 \\
bf & 4,363 & 4,380 & 4,356 & 4,367 & 20,005 \\
b5 & 4,789 & 4,774 & 4,693 & 4,752 & 02,914 \\
ba & 4,588 & 4,578 & 4,512 & 4,559 & 04,508 \\
be & 4,596 & 4,571 & 4,503 & 4,557 & 09,992 \\
\hline
\end{tabular}

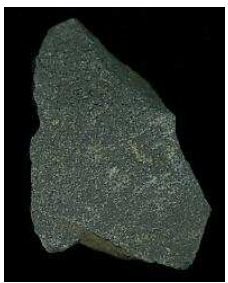

(a) b

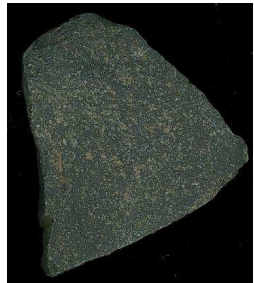

(b) br

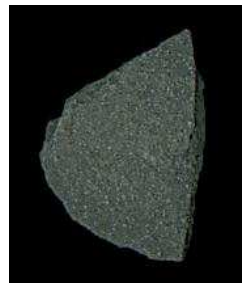

(c) bf

Figura 6. Exemplos de imagens originais coloridas e sem equalização de partículas representativas das amostras basálticas sem alteração

classificação automático que atualize e/ou crie novas subclasses, caso necessário, em função da incorporação de um novo agregado analisado.

Este artigo propõe também uma abordagem de classificação automática que utiliza como entrada valores de entropia dos agregados, expressos através de vetores característicos compostos de 3 valores e gera como saída classes que representam agregados alterados e não alterados. Esses valores representam a entropia obtida para cada componente RGB da imagem digital:

$$
S^{i}=\left[\begin{array}{lll}
x_{1}^{i} & x_{2}^{i} & x_{3}^{i}
\end{array}\right]^{T}
$$

sendo que $S^{i}$ representa um vetor característico $i$ e os valores de $x_{j}^{i}$ correspondem às características (atributos) deste vetor. As amostras obtidas compõem um conjunto $T$ :

$$
T=\left\{S^{1}, S^{2}, \ldots, S^{n}\right\}
$$

O conjunto $T$ define todos os agregados obtidos em campo, através de seus vetores característicos. 


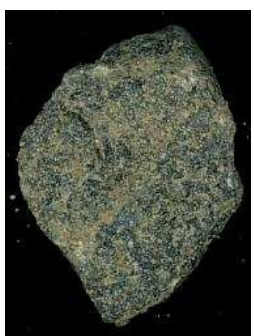

(a) b5

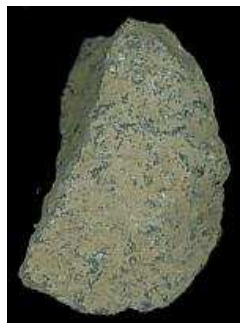

(b) ba

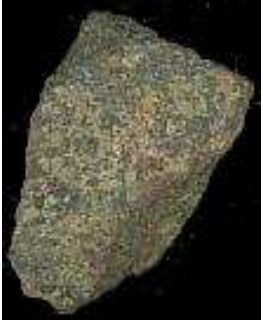

(c) be

Figura 7. Exemplos de imagens originais coloridas e sem equalização de partículas representativas das amostras basálticas alteradas

A abordagem de classificação é organizada em duas etapas. Na primeira etapa, as amostras são classificadas em alteradas e em não alteradas. Na segunda etapa, as amostras alteradas são organizadas em grupos, de acordo com o nível de alteração. A organização em duas etapas permite que a classificação seja mais sensível às diferenças do estado de alteração dos agregados, por inicialmente separar as amostras em dois grupos. Tais etapas são descritas nas subseções a seguir.

\subsection{Classificação dos Agregados quanto à Alteração}

Nessa etapa, é importante que o algoritmo consiga diferenciar as amostras em dois grupos principais: alterados e não alterados. A arquitetura de redes neurais ART 2A-E $[8,28]$ é empregada para realizar a classificação das amostras. Essa arquitetura representa um conjunto de redes neurais auto-organizáveis e geralmente de aprendizado de máquina não supervisionado que é capaz de aprender códigos de representação em resposta a uma sequiência arbitrária de padrões de entrada [6,7]. De maneira geral, tais redes encontram de maneira rápida vetores de dimensão (número de atributos) igual ao padrão de entrada, sendo que cada vetor representa um agrupamento (cluster) de dados. Assim, cada agrupamento corresponde a uma unidade de representação de dados.

As redes ART diferem das demais arquiteturas auto-organizáveis por permitir que o usuário controle o grau de similaridade entre os padrões agrupados em um mesma unidade de representação. Esse controle permite que a rede seja mais ou menos sensível às diferenças que existem nos padrões de entrada, gerando assim mais ou menos agrupamentos de acordo com esse controle.

O aprendizado nas redes ART é tratado como uma atividade contínua e não estática como em outras redes neurais, por exemplo a rede de perceptrons de múltiplas camadas 
(MLP). Tal forma de aprendizado permite que a rede adapte-se de maneira automática a novos padrões de entrada, criando, se necessário, novas unidades para representar tais padrões.

A organização da rede ART 2A-E corresponde de maneira geral a arquitetura ART tradicional [7]. Essa arquitetura é organizada através de dois sistemas principais: atenção e orientação (Figura 8). O sistema de atenção tem 3 camadas de neurônios: camada de pré-processamento $F_{0}$, camada de representação das entradas $F_{1}$ e camada das unidades de representação $F_{2}$. As camadas, com exceção da camada $F_{2}$, tem quantidade de neurônios igual à quantidade de atributos dos padrões de entrada. A camada $F_{2}$ inicialmente não apresenta neurônios. Neurônios são adicionados a esta camada (característica auto-organizável) à medida que padrões de entrada são apresentados e representados pela rede.

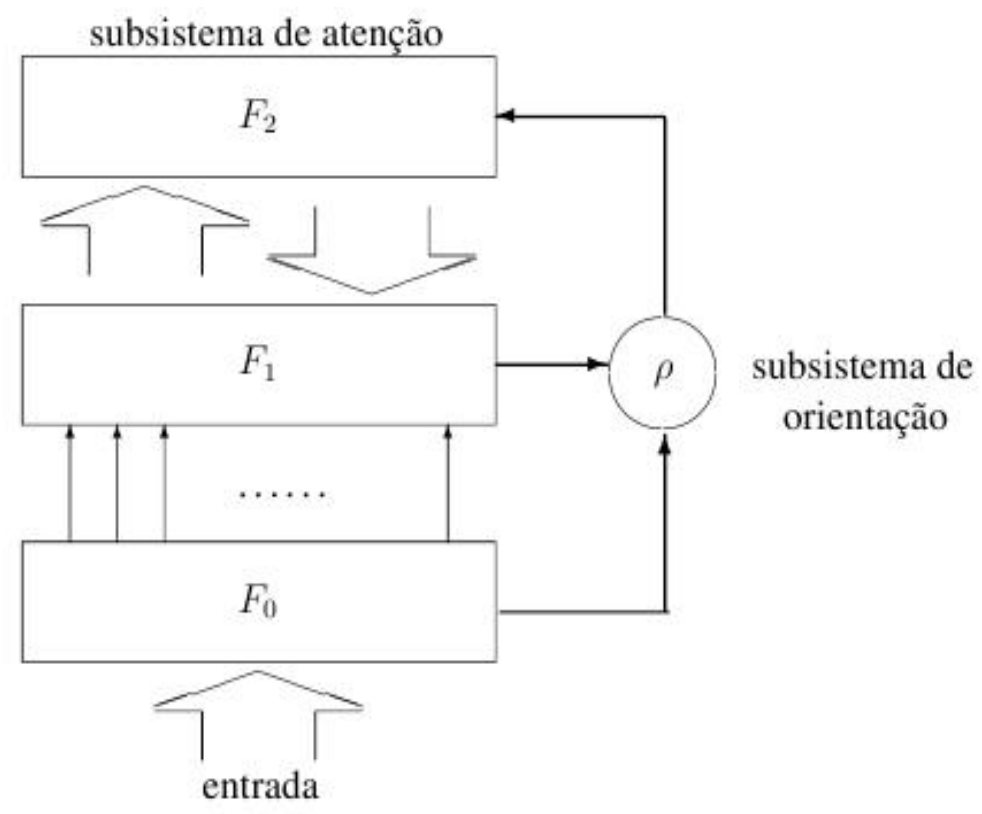

Figura 8. Organização básica das redes neurais ART

A camada $F_{1}$ e a camada $F_{2}$ são interconectadas através de um conjunto de pesos adaptativos chamados de bottom-up $\left(F_{1} \rightarrow F_{2}\right)$ e top-down $\left(F_{2} \rightarrow F_{1}\right)$. O caminho a partir do neurônio $i$ da camada $F_{1}$ para o neurônio $j$ da camada $F_{2}$ é representado por $w_{i j}$. Similarmente, o neurônio $j$ da camada $F_{2}$ é conectado com o $i$ da camada $F_{1}$ através dos pesos $w_{j i}$. Os pesos multiplicam os sinais que são enviados entre as camadas de neurônios e são responsáveis principalmente pelo armazenamento do conhecimento. A interação entre as 
camadas $F_{1}$ e $F_{2}$ é controlada pelo sistema de orientação. Esse sistema emprega o parâmetro de vigilância $(\rho)$ e atua de acordo com o algoritmo de treinamento (Algoritmo 1).

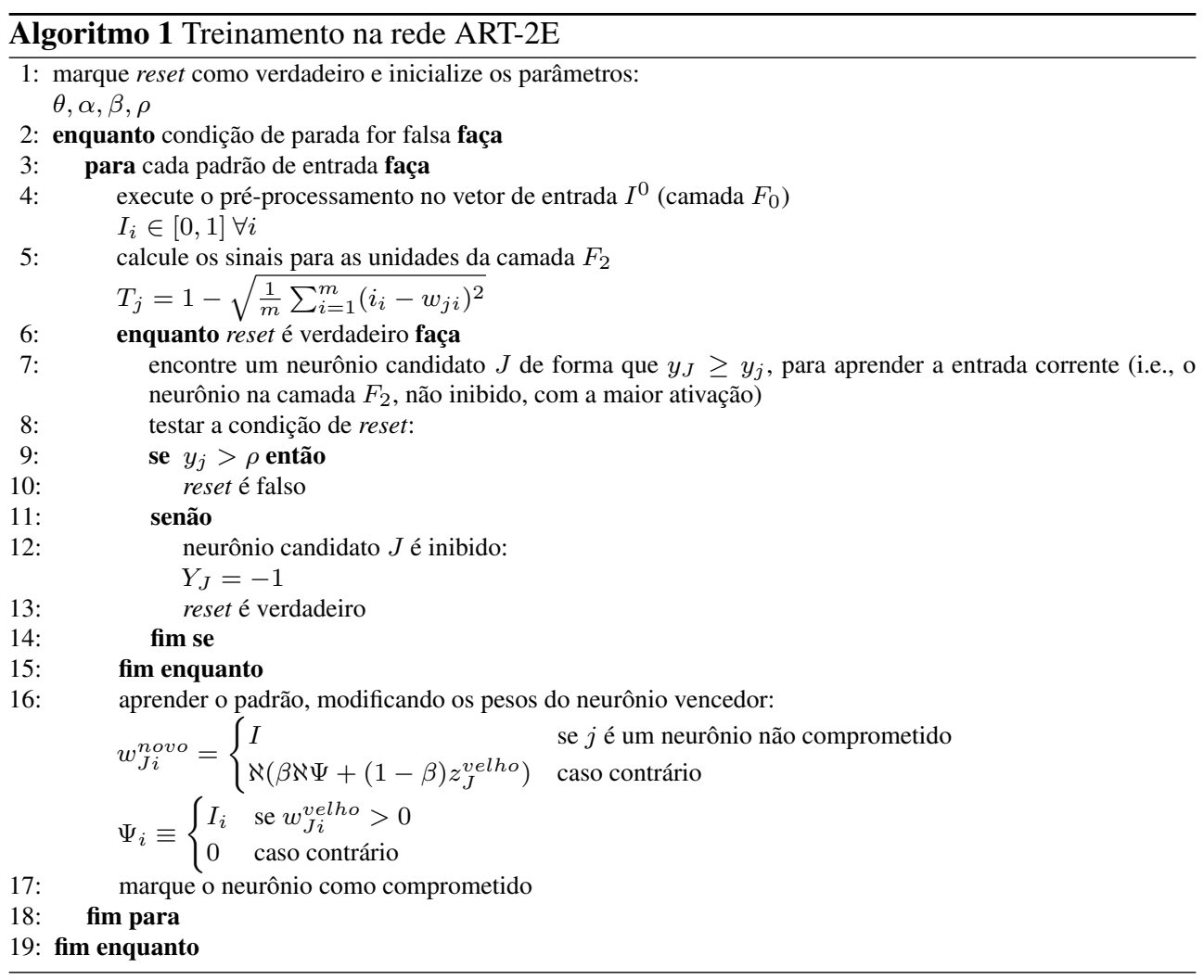

Inicialmente, na camada $F_{0}$, os elementos do vetor de entrada são normalizados no intervalo $[0,1]$ (Linha 4). Tais elementos servem como entrada para a camada $F_{1}$, responsável por atualizar a ativação dos neurônios da camada de representação $F_{2}$ (Linha 5). A distância euclidiana entre o padrão corrente e o conjunto de pesos é calculada e subtraída de 1 , de forma a garantir que um padrão de entrada idêntico ao conjunto de pesos $w_{j i}$ produza uma distância igual a 1 .

Após o cálculo de ativação dos neurônios da camada de representação, a rede inicia a etapa de busca, que visa encontrar o neurônio candidato $J$ de maior ativação dessa camada (Linha 6). A ressonância e a adaptação ocorrem quando o candidato $J$ é um neurônio não comprometido (i.e., não foi previamente escolhido para aprender outro padrão), ou se a ativação do neurônio candidato $T_{J}$ for menor ou igual ao parâmetro de vigilância $\rho$. Na 
prática, a definição de neurônio não comprometido só é válida se o algoritmo trabalhar com uma representação fixa (estática) de neurônios na camada $F_{2}$. Em uma implementação dinâmica, inicialmente não existem neurônios na camada $F_{2}$. Neurônios são criados sob demanda do algoritmo para representar novos padrões de entrada.

As constantes $\alpha, \beta$ e principalmente o valor de vigilância $\rho$ orientam o processo de aprendizado. A constante $\alpha$ ou parâmetro de escolha define a profundidade máxima de busca por um neurônio candidato. Com $\alpha=0$, todos os neurônios da camada $F_{2}$ são verificados antes que um neurônio comprometido seja escolhido como vencedor. $\mathrm{O}$ valor dessa constante deve ser escolhido obedecendo-se o intervalo $0 \leq \alpha \leq \frac{1}{\sqrt{m}}$.

O parâmetro $\beta$ descreve a taxa de aprendizado da rede. Tal parâmetro deve respeitar o intervalo $0 \leq \beta \leq 1$. Com $\beta=0$, a rede opera no modo de classificação. Nesse modo, a rede devolve um neurônio vencedor $J$, mas os pesos não são atualizados, de forma que o padrão apresentado não é aprendido pela rede. Esse modo de operação é interessante quando busca-se a classificação de um conjunto de valores de entrada sem que exista a atualização da rede. Com $\beta=1$, a rede opera no modo de aprendizado rápido, onde o vetor de pesos do neurônio $J$ é substituído, sem adaptação, ao padrão de entrada. Valores dentro do intervalo $0<\beta<1$ fazem com que a rede opere no modo de aprendizado lento, de forma que os pesos sejam gradativamente adaptados aos padrões de entrada. Valores de $\beta$ mais próximos de 0 representam uma adaptação mais suave, enquanto valores de $\beta$ mais próximos de 1 reproduzem uma adaptação mais agressiva. $\mathrm{O}$ valor do parâmetro $\beta$ pode variar durante a apresentação de um conjunto de padrões, dependendo do número de ciclos de treinamento. Esse valor não deve ser usado na forma $\beta \cong 1$, pois os protótipos tendem a desviar entre os padrões de entrada ao invés de convergir para uma média dos padrões agrupados na classe.

O parâmetro de vigilância $\rho$ define o número de agrupamentos que serão criados pela rede. A principal diferença da rede ART 2A-E em relação as redes ART tradicionais consiste na utilização da distância Euclidiana ao invés do cosseno do ângulo formado da entrada com o vetor de protótipos [12]. Na rede ART-2A, o valor desse parâmetro forma uma fronteira de decisão circular de raio igual a $\sqrt{2(1-\rho)}$ em torno do vetor de protótipos de cada grupo [15]. Isso permite que a escala dos valores de entrada seja preservada, por não empregar a normalização euclidiana nos vetores de entrada. $\operatorname{Com} \rho=0$, todos os padrões de entrada são agrupados no mesmo grupo. Com $\rho=1$, é criado um grupo para cada padrão distinto de entrada apresentado à rede.

Pode-se observar que rótulos não são empregados na classificação. Na prática, são empregadas medidas de desempenho de agrupamento (clustering) adaptadas para a organização da rede ART 2A-E para verificar a qualidade da classificação em relação as valores dos atributos de entrada dos padrões. Duas medidas de desempenho são empregadas para avaliar a qualidade da classificação de dados obtido. A primeira medida utilizada é a distância intra definida pela Equação 10. Essa medida permite quantificar a distância média entre padrões 
contidos em uma classe $i$ e seu centróide $c_{i}$, empregando uma função de distância $d(A, B)$ :

$$
\text { Intra }=\frac{\sum_{i=1}^{m} \sum_{j=1}^{n} d\left(c_{i}, x_{j}\right)}{m}
$$

onde $m$ é o número de classes formadas pela rede, $x_{j}$ é o padrão corrente e $n$ o tamanho do vetor de entrada, que para este trabalho é igual a 3.

A segunda medida, chamada de inter, mede a distância entre os centróides das classes criadas pela rede ART 2A-E:

$$
\text { Inter }=\frac{\sum_{i=1}^{m} \sum_{j=1}^{m} d\left(c_{i}, c_{j}\right) \forall i \neq j}{m}
$$

Essa medida permite observar o quanto as classes, representadas pelo valor do centróide, diferem entre si. A função de distância $d(A, B)$ usadas nas medidas intra e inter é definida por:

$$
d(A, B)=\frac{A \cdot B}{\|A\| \cdot\|B\|}
$$

Empregando essas medidas, os resultados podem ser plotados na mesma escala, permitindo definir um ponto no plano $R^{2}$ onde ambas as funções sejam igualadas, isto é, onde a distância intra é minimizada enquanto a inter é maximizada (Figura 6.1). Regiões próximas ao ponto de encontro freqüentemente definem o valor de $\rho$ mais adequado ${ }^{3}$ e, conseqüentemente, o número de classes e padrões por classes gerados pela ART 2A-E.

Os resultados obtidos com a rede ART 2A-E estão relacionados na Tabela 4. Os resultados estão expressos pelo erro obtido na classificação, empregando validação cruzada. Assim, quanto menor o valor do erro de classificação obtido, melhor o desempenho de agrupamento/classificação do algoritmo. A classificação é realizada em duas classes: agregados não alterados e agregados alterados. Tais resultados são comparados com outros algoritmos comumente utilizados para o agrupamento e classificação de dados. Os algoritmos estão organizados em dois grupos: agrupadores e classificadores. Os agrupadores extraem conhecimento dos dados sem utilizar a rotulação (ou classificação prévia realizada em campo) das amostras. Os algoritmos desse grupo são: KMeans [20], Density Based Clusterer [4], CobWeb [11], Expectation-maximization clustering (EM) [31]. Nesse grupo, os rótulos são empregados nos agrupadores apenas para a avaliação dos agrupamentos obtidos e cálculo do erro de classificação. A rede ART 2A-E é inserida nesse grupo por igualmente não empregar os rótulos para conduzir o aprendizado.

\footnotetext{
${ }^{3} \mathrm{O}$ valor de $\rho$ da intersecção é mais adequado por permitir que o número de classes seja minimizado sem que padrões diferentes sejam categorizados em um mesmo agrupamento
} 


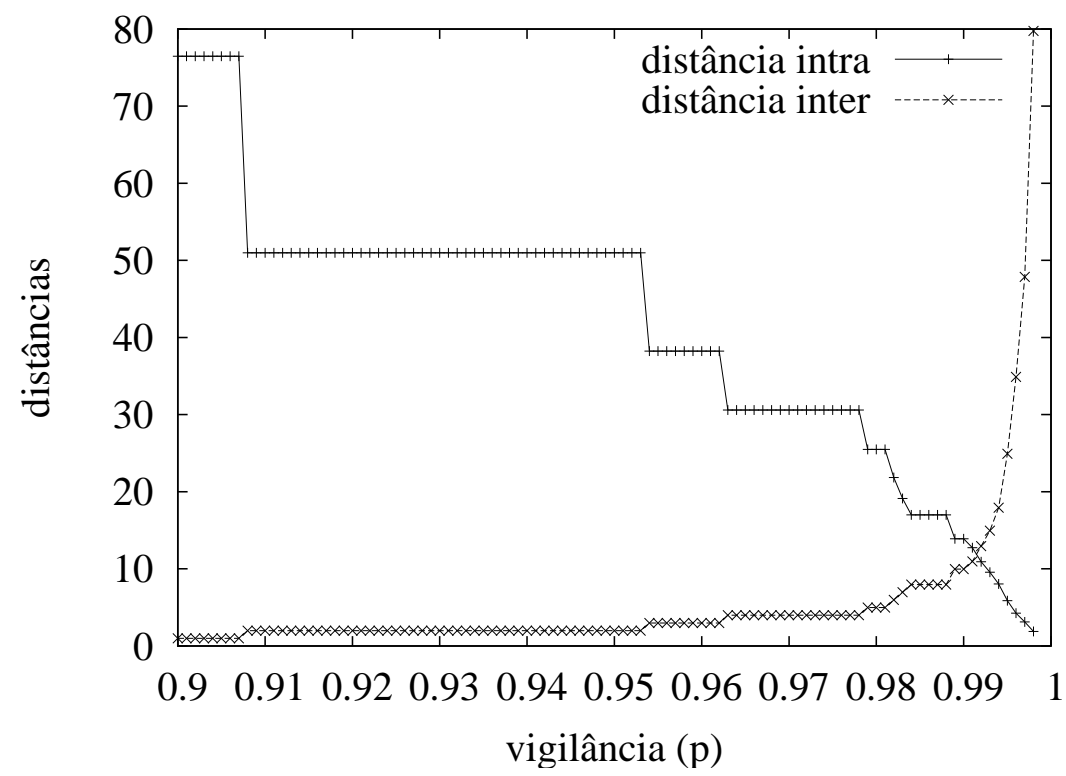

Figura 9. Valores de distância para diferentes valores de vigilância $(\rho)$

Os classificadores realizam o aprendizado supervisionado empregando os rótulos para guiar o processo de aprendizado. Os algoritmos desse grupo são: Multi layer perceptrons (MLP) [14], Naive Bayes [17], Locally Weighted Learning (LWL) [1], Bayesian networks [16], J48 (Weka C4.5 implementation) [25].

A rede ART 2A-E apresenta o melhor resultado em relação ao erro de classificação obtido mesmo quando comparada com abordagens supervisionadas, que além do valor de entropia das amostras, empregam a classificação prévia realizada em campo. Logo, a capacidade da rede em extrair conhecimento a partir das características dos agregados é notável. A rede neural de perceptron multi camadas teve desempenho similar, mas sofre do problema de falta de estabilidade e plasticidade na aquisição de conhecimento [5].

Além disso, a abordagem de classificação através da rede ART 2A-E realiza o aprendizado fundamentalmente incremental, ao contrário das outras abordagens (com exceção do algoritmo LWL). Isso permite que novas amostras de campo possam ser inseridas no modelo e o conhecimento seja estendido sem a perda do conhecimento previamente obtido. Tal característica é altamente desejável para a solução do problema em questão, que tem natureza dinâmica. 
Tabela 4. Classificação das amostras

\begin{tabular}{llccc}
\hline Grupo & Algoritmo & Supervisionado & Incremental Erro (\%) \\
\hline \multirow{5}{*}{ Agrupadores } & ART 2A-E & não & sim & 07,84 \\
& Kmeans & não & não & 14,37 \\
& Density & não & não & 14,38 \\
& CobWeb & não & não & 50,00 \\
& EM & não & não & 83,00 \\
\hline \multirow{4}{*}{ Classificadore } & MLP & sim & não & 11,95 \\
& Naive Bayes & sim & não & 13,04 \\
& LWL & sim & sim & 13,39 \\
& Bayes Net & sim & não & 14,05 \\
& J48 & sim & não & 14,38 \\
\hline
\end{tabular}

\subsection{Classificação de Agregados Alterados}

Nessa etapa, os agregados classificados como alterados são utilizados como um novo conjunto de treinamento. Os resultados da comparação dos algoritmos estão apresentados na Tabela 5 .

Os resultados em relação ao erro para os classificadores foram agora organizados em dois valores, separados por uma barra (first/second). O primeiro valor indica o erro de classificação empregando a rotulação existente para as amostras. Considerando esse erro, pode-se observar que os classificadores tiveram o pior desempenho na classificação. Isso se deve ao fato dos algoritmos tentarem empregar os rótulos relacionados com a origem do material para conduzir a classificação, ao invés de concentrarem-se apenas nas características intrínsecas dos dados de entrada. Por empregar a rotulação, os algoritmos não conseguem separar as amostras dos grupos ba e be, notavelmente próximas em escala (Figura 7), o que eleva (próximo a 40\%) o erro de classificação.

O segundo valor de erro indica uma situação onde os grupos ba e be são rotulados previamente em apenas um grupo, antes de serem processados pelo algoritmo. Apesar de não viável em situações práticas, essa rotulação prévia permite que os classificadores separem as amostras em grupos, fato que não ocorreu quando foram empregadas as amostras com a rotulação inicial.

Novamente, considerando a flexibilidade da abordagem de classificação e o valor do erro (que foi observado próximo a 8\%), a rede ART 2A-E apresentou o melhor desempenho na classificação. Isso define que nas duas fases, o melhor algoritmo de classificação observado foi a rede ART 2A-E. 
Tabela 5. Classificação das amostras

\begin{tabular}{llccc}
\hline Grupo & Algoritmo & Supervisionado Incremental & Erro (\%) \\
\hline \multirow{5}{*}{ Agrupadores } & ART 2A-E & não & sim & 07,86 \\
& Kmeans & não & não & 09,15 \\
& Density & não & não & 09,15 \\
& CobWeb & não & não & 33,34 \\
& EM & não & não & 58,16 \\
\hline \multirow{5}{*}{ Classificadores } & MLP & sim & não & $49,05 \mid 09,43$ \\
& Naive Bayes & sim & não & $36,60 \mid 07,54$ \\
& BWL & sim & sim & $43,39 \mid 09,43$ \\
& Jayes Net & sim & não & $43,39 \mid 07,54$ \\
& J48 & sim & não & $49,05 \mid 09,43$ \\
\hline
\end{tabular}

\section{Conclusões}

O agregado mineral é utilizado como constituinte principal de muitos materiais usados para o propósito de construção de obras e uma de suas principais exigências em quase todas as circunstâncias de uso é que sejam fortes e duráveis. Essas características variam muito quanto ao estado de alteração dos agregados. Agregados em estados alterados geralmente têm sua durabilidade e resistência mecânica comprometidas, vindo a comprometer a capacidade estrutural e mecânica da estrutura por ele formada.

A entropia foi empregada como um novo método de análise do estado e níveis de alteração dos agregados. Verificou-se que a entropia das imagens coloridas e sem equalização foi capaz de diferenciar todos os agregados alterados daqueles não alterados, assim como foi sensível para diagnosticar níveis de alteração diferentes entre os agregados alterados. Tal diferenciação entre os níveis de alteração permite que sejam obtidos resultados quantitativos, qualitativos e não subjetivos.

O método de classificação automática proposto facilita o processo de analise dos estados e níveis de alteração e torna possível atualizar e/ou criar novas subclasses de níveis de alteração em função de incorporação de novas amostras de agregados. A abordagem de classificação através da rede neural ART 2A-E mostrou-se adequada ao problema, com um erro de classificação menor quando comparada com outras abordagens. Isso permite a construção de um modelo de conhecimento que permite diferenciar, de maneira automática, amostras de agregados alterados de não alterados, com a possibilidade de extensão desse conhecimento a partir da análise de novas amostras. 
Uma avaliação do estado de alteração de maneira rápida e eficiente como no método apresentado é de grande importância para todo o setor de consumo de agregados minerais, assim como para as pedreiras que poderão avaliar rapidamente a qualidade de seu material fornecido. Nesse caso, uma possível forma de utilização do método é durante a produção do agregado, com a captura de imagens de amostras obtidas na esteira, possibilitando a interrupção do processo produtivo caso amostras alteradas sejam detectadas.

O método de análise e classificação do estado e níveis de alteração dos agregados através da entropia mostra-se promissor, no entanto, mais estudos devem ser realizados com um maior numero de amostras basálticas, assim como ampliar os estudos para outros tipos de agregados.

\section{Agradecimentos}

Luciano José Senger e Lilian Taís de Gouveia agradecem a Fundação Araucária pelo suporte. Lilian Taís de Gouveia também agradece à FAPESP (2007/01128-0) pelo suporte financeiro. Os autores agradecem também aos comentários valiosos dos revisores desta revista.

\section{Referências}

[1] Christopher G. Atkeson, Andrew W. Moore, and Stefan Schaal. Locally weighted learning for control. Artificial Intelligence Review, 11(1-5):75-113, 1997.

[2] N. S. Baer and R. Snethlage. Group report: What is the state of our knowledge of the mechanisms of deterioration and how good are our estimates of rates of deterioration? In Saving our Architectural Heritage: The Conservation of Historic Stone Structures, pages 95 - 112. John Wiley \& Sons Ltd., Berlin, 1996.

[3] F. G. Bartley, G. Bignall, C. C. Harvey, A. B. Christie, A. Reyes, R. Soong, and K. Faure. Clay mineralogy of modified marginal aggregates. Technical Report 318, Transfund New Zealand Research Report, 2007.

[4] S. Brecheisen, H. Kriegel, and M. Pfeifle. Multi-step density-based clustering. 9(3), 2006.

[5] G. A. Carpenter, M. N. Gjaja, S. Gopal, and C. E. Woodcock. ART neural networks for remote sensing: Vegetation classification from lansat TM and terrain data. IEEE Transactions on Geoscience and Remote Sensing, 35(2), March 1997.

[6] G. A. Carpenter and S. Grossberg. The ART of adaptive pattern recognition by a self-organizing neural network. Computer, 21(3):77-88, Março 1988. 
[7] G. A. Carpenter and S. Grossberg. ART 2: Self-organization of Stable Category Recognition Codes for Analog Input Patterns. Applied Optics, 26(23):4919-4930, 1989.

[8] G. A. Carpenter, S. Grossberg, and D. B. Rosen. ART 2-A: An Adaptive Resonance Algorithm for Rapid Category Learning and Recognition. Neural Networks, 4:494-504, 1991.

[9] Luciano Fontoura Costa and Roberto Marcondes Cesar Jr. Shape Analysis and Classification. CRC Press, 2001.

[10] Edward R. Dougherty and Roberto A. Lotufo. Hands-on Morphological Image Processing. SPIE PRESS, 2003.

[11] Doug Fisher. Iterative optimization and simplification of hierarchical clusterings. Technical Report Technical Report CS-95-01, 2005.

[12] T. Frank, K. Kraiss, and T. Kuhlen. Comparative analysis of fuzzy art and art-2a network clustering performance. IEEE transactions on neural networks, 9(3):544-559, 1998.

[13] C. C. Gifkins, W Herrmann, and R. R. Large. Altered Volcanic Rocks: A Guide to Description and Interpretation. Hobart: Centre for Ore Deposit Research, Tasmania, 2005.

[14] S. Haykin. Neural Networks - A Compreensive Foundation. Prentice-Hall, 1994.

[15] Ji He, Ah-Hwee Tan, and Chew-Lim Tan. Modified art 2a growing network capable of generating a fixed number of nodes. IEEE Transactions on Neural Networks, 3(15):728-737, 2003.

[16] David Heckerman, Dan Geiger, and David M. Chickering. Learning bayesian networks: The combination of knowledge and statistical data. In Machine Learning, pages 197-243, 1995.

[17] George H. John and Pat Langley. Estimating continuous distributions in bayesian classifiers. In Proceedings of the Eleventh Conference on Uncertainty in Artificial Intelligence, pages 338-345, San Mateo, 1995. Morgan Kaufmann.

[18] Geoffrey Lees and Christopher K. Kennedy. Quality, shape and degradation of aggregates. Quarterly Journal of Engineering Geology and Hydrogeology, 8:193 $209,1975$.

[19] Arlei Benedito Macedo. Recursos minerais não-metálicos. Estudos Avançados, 12(33), 1998. 
[20] Y. Marzouk and A. Ghoniem. K-means clustering for optimal partitioning and dynamic load balancing of parallel hierarchical N-body simulations. Journal of Computational Physics, 207(2):493-528, 2005.

[21] F. Murtagh, X. Qiao, P. Walsh, P.A.M. Basheer, D. Crookes, and A. Long. Grading of construction aggregate through machine vision: results and prospects. Computers in Industry, 56:905 - 917, 2005.

[22] A. M. S. Oliveira and S. N. A. Brito. Mgeologia de engenharia. Technical report, Associação Brasileira de Geologia de Engenharia - ABGE, São Paulo, 1998.

[23] Helio Pedrini and Willian Robson Schwartz. Análise de Imagens Digitais - Princípios, Algoritmos e Aplicações. Thomson, 2008.

[24] M.I. Pinard and P.Jackalas. Durability aspects of chemically stabilized, weathered basaltic materials for low-volume road base construction. Transportation Research Record, 1, 1987.

[25] J. R. Quinlan. Improved use of continuous attributes in c4.5. Journal of Artificial Intelligence Research, (4):77-90, 1996.

[26] F. L. Roberts, P. S. Kandhal, E. R. Brown, D. Y. Lee, and T. W. Kennedy. Hot mix asphalt materials, mixture design, and construction. NAPA Education Foundation, Maryland, 1996.

[27] W. C. Schefler. Statistics: Concepts and Applications. The Benjamin/Cummings Publishing Company, 1988.

[28] L. J. Senger. Escalonamento de processos: uma abordagem dinâmica e incremental para a exploração de características de aplicações paralelas. PhD thesis, ICMC-USP, Feb. 2005.

[29] C. E. Shannon. A mathematical theory of communication. Bell System Technical Journal, 27:379 - 423, 1948.

[30] Arezki Tagnit-Hamou, Mladenka Saric-Coric, and Patrice Rivard. Internal deterioration of concrete by the oxidation of pyrrhotitic aggregates. Cement and Concrete Research, 35:99 - 107, 2005.

[31] Ian H. Witten and Eibe Frank. Data Mining: Practical Machine Learning Tools and Techniques, Second Edition (Morgan Kaufmann Series in Data Management Systems). Morgan Kaufmann, June 2005. 